\title{
The death of powder - micro-electron diffraction with EIGER
}

\author{
Clemens Schulze-Briese ${ }^{a}$, Andreas Förster ${ }^{b}$, Pascal Hofer $^{c}$, Sacha De Carlo ${ }^{d}$, Luca Piazza ${ }^{e}$, \\ Julian TC Wennmacher ${ }^{f}$ and Tim Grüne ${ }^{g}$. \\ ${ }^{a}$ DECTRIS Ltd., Täfernweg 1, 5405 Baden-Dättwil, Switzerland, clemens.schulze-briese@dectris.com \\ ${ }^{b}$ DECTRIS Ltd., Täfernweg 1, 5405 Baden-Dättwil, Switzerland, andreas.foerster@dectris.com \\ 'DECTRIS USA Inc., 325 Chestnut Street, Suite 800, Philadelphia, PA, USA, pascal.hofer@dectris.com \\ ${ }^{d}$ DECTRIS Ltd., Täfernweg 1, 5405 Baden-Dättwil, Switzerland, sacha.decarlo@dectris.com \\ eDECTRIS Ltd., Täfernweg 1, 5405 Baden-Dättwil, Switzerland, luca.piazza@dectris.com \\ fDepartment of Energy and Environment, Paul Scherrer Institute, Forschungsstrasse 111, 5232 \\ Villigen, Switzerland, julian.wennmacher@psi.ch \\ ${ }^{\mathrm{g}}$ Faculty of Chemistry, University of Vienna, Währinger Straße 42, 1090 Wien, Austria, \\ Tim.Gruene@univie.ac.at
}

X-ray crystallography is the method of choice to obtain the atomic coordinates of synthesized or isolated molecules, such as drug candidates. The major bottleneck for determining crystal structures is the existence of single crystals of sufficient size and quality. Many synthesized compounds fail to form large single crystals even after crystallization experiments in various solvents. Chemists are often left with powders unsuitable for structural studies by X-ray crystallography.

Most powders are not amorphous but microcrystalline. Chemical research and pharmaceutical development would progress faster if structures could be determined reliably at high resolution from microcrystals. Electron diffraction promises to do just that, primarily because electrons interact with matter much more strongly than photons. However, current electron microscopes are not optimized for diffraction. The sample stage is a poor substitute for a goniometer, and traditional detectors suffer from noise and low dynamic range.

Here we show that a mid-range microscope equipped with a photon-counting direct detector is an excellent tool to determine atomic structures from microcrystals with the smallest dimension of a few hundred nanometers. We determined the structure of a new methylene blue derivative at $0.9 \AA$ resolution. Highlighting the potential of electron diffraction for the pharmaceutical industry, we also determined the structure of the active component of a commercially available drug from material taken from the capsule.

The key factor for high data collection quality and speed was a very fast and sensitive hybrid photon-counting detector, which allowed structures to be determined in a few hours. Technical developments, especially on the microscope, will make this process even easier and more straightforward. In summary, we demonstrate the potential of electron diffraction to transform industries that rely on the atomic structure of small compounds, such as drug discovery, formulation and preparation. 